I. To the municipality, it offers relief from all care of the cases in question, and without cost for horses, ambulance, or treatment.

2. To the hospital surgeons, it supplies the kind of cases especially desired by those who are teachers.

3. To the patients, it secures prompt help, and the most skilful attendance.

The municipal element in the system maintains in all concerned the consciousness of accountability, while the voluntary element in the system quickens between the several hospitals a lively emulation. Take it all in all, the ambulance system of New York is creditable, both to the medical and to the municipal authorities of the city, presenting an unique example of an efficient and harmonious combination of municipal and voluntary effort, which seems to yield satisfaction to all concerned.

As the city which, for the protection of property, boasts the best fire department in the world, must sooner or later follow its higher instincts and traditions in having a system correspondingly complete for the protection and care of human life also, it is possible that some of the above facts will afford memoranda which may be of service in the organisation of the future Ambulance Department of London.

\section{THE USE OF FLECTRICITY IN EAR-DISEASE.*}

$$
\text { By E. WOAKES, M.D. }
$$

IN discussing the subject of the use of electricity in diseases of the ear, I shall refer to it-first, as a means of diagnosis; and secondly, as a remedial agent.

As regards the value of electricity for the purposes of diagnosis in ear-disease, you will at once recall the experiments of Dr. Brenner at St. Petersburg, first made known about twelve years ago by the publication of his work on Electro-Otiatrics, a very fair résumé of which is given in Dr. Burnett's treatise on the Ear, and also in Dr. Roosa's last edition. You will remember that this work was the result of ten years of careful study, and therefore the conclusions arrived at are entitled to the weight which such exhaustive examination by a competent observer must always demand. The outcome of this study was, that the auditory nerve could be excited by the electric current, which in the normal state responded to it by certain constant phenomena; and that these phenomena were reduced to exact formulæ for the guidance of others, who would ascertain by means of electricity the state of the nerve of audition.

That Brenner's conclusions should meet with opposition, is only what may have been expected. Without desiring to prejudice the discussion, I shall state very briefly two points which seem to my mind fatal to the sufficiency of the experiments, and therefore to the conclusions deduced from them.

The conditions of the experiment make it probable that the auditory nerve is not reached by the current at all. The reasons for this conclusion are, that, when a nerve is superficially placed, covered only by integument-i.c., when the electrode is applied to it-a very considerable portion of the current is diffused in the surrounding tissues. Bone being a bad conductor, this loss of electricity, de Watteville and Hitzig argue, not taking place, increases the probability of the current reaching the auditory nerve. (The electrode is placed in the external canal, previously filled with water.) But the current must previously affect other nerves within reach-the branches of the fifth-and reflexly excite spasm of the tensor tympani, before it reaches the auditory nerve. Now, the effect of exciting a powerful contraction of the tensor tympani is to make pressure through the stapes on the intralabyrinthine fluid. Dr. Weber-Liel of Berlin has pointed out to me that direct pressure over the stapes, when this bone is exposed by means of a probe, is accompanied by a sound which the patient experiences, resembling "ting-g-g". The correspondence between this sound and that produced by Brenner, and understood by him to imply a normal reaction to galvanism of the auditory nerve, is very significant. At any rate, it leaves one's mind in a state of doubt as to whether Brenner's normal reaction was not the result of inducing contraction of the tensor tympani in a healthy ear, seeing that such contraction, when in excess of what occurs in response to ordinary stimuli, is undoubtedly associated with a sound; that is to say, the sound is clue in both cases to mechanical pressure, and not to electric excitation of the auditory nerve.

Another point, which stands somewhat in the light of a corollary to the preceding, is this: the supporters of Brenner's hypothesis attach great importance to the fact that, when the test is applied to a badly

* Read at Cambridge to introduce the discussion on this subject at the Otological Section, August re8o. diseased ear of long standing, the phenomena do not occur in that ear, but in the opposite one, to which no electricity is applied; or they occur in both ears in a reverse order. The current is here supposed to pass along the partially paralysed auditory nerve of the diseased side, and to be transferred to the other and unaffected nerve by communications at their origin. Now I think there is another explanation, and a more reasonable one. It is very rare for an old-standing case of eardisease to be free from paresis of the tube-muscles on the affected side. Now, if the conclusion at which I have already arrived hold goodviz., that the sound in question is the result of contraction of the tensor tympani-it is very obvious that, if this muscle be paralysed, or only partially paralysed, on the side to which the galvanism is applied, it will fail to react to the stimulus, or will react partially, or differently from what it would do otherwise. It is not improbable, under these circumstances, that the current would travel to the opposite branches of the fifth nerve; and that contraction of the tensor tympani of the opposite ear would ensue, and give the ordinary result in this, the unaffected side. That such cross action of a stimulus in the case of the fifth nerve does occur, is borne out by the following suggestive fact. I lady about fifty years of age, suffering with deafness and paresis of the tubal muscles of the right ear, not of an extreme type, states that, when she bites a crust or hard substance on her left upper second bicuspid tooth, she gets a distinct momentary ringing sound in her right ear. She is an intelligent common-sense person, not at all likely to imagine the symptom; and the statement I have repeatcl was volunteered to me. I need not stay to point out that the connection here can only be through the fifth nerve, and that it was transferred to the opposite side; thus establishing, as far as it goes, the possibility of a sound being excited through this nerve in the opposite ear.

"Professor Hughes's audiometer is an instrument for exactly measuring the power of hearing, and chronicling the progress of recovery from deafness." In the Practitioner for May 1880, Dr. Richardson is reported to affirm that it "may be used to differentiate between deafness through the external ear and deafness from closure of the Eustachian tube-throat-deafness; or to determine the value of artificial tympanums in instances of deafness due to imperfection or destruction of the natural tympanum." Others speak of it as an expensive and im. posing toy. I cannot say that I am in a position to throw any light upon these extreme views, though I trust the discussion may do so.

I now have to consider the value of electricity in ear-disease, considered as a curative agent simply. I do not know how far the experience of other otologists corresponds in this respect with my own, but I am quite free to confess to a growing recognition of the paramount import of muscle-paresis as a chief factor in the causation of deafness and its concomitant symptoms in a very large proportion of those cases of the disease which occur in adult life. To Dr. Weber-Liel of Berlin is undoubtedly due the credit of having first poirted out this important fact in his work entitled Progressize Schzorhöri kcit, published in 1873. Three years ago, before I became acquainted with this work, I had quite independently arrived at the conclusion that paresis of muscle was in some way concerned in the production of the symptoms referred to, because I met with quite a number of cases in which these symptoms were associated with marked paralysis of the palato-tubal muscles. It is true that most of my cases presented some marked divergencies, as regards simply aural conditions, to those described by Weber-Liel; and I presented the first rísumí of them to this Section in a paper which I had the honour of submitting to it last year at Cork. It would be beside my duty on this occasion to occupy your time with further details on this subject; suffice it to say that there appear to me two distinct phases of paralytic affections of the atilitory apparatus-viz., that described as "progressive" deafness by Weber-Licl, and that which I have elsewhere described as "paretic" dealness. Now the importance to our present purpose of these observations, reciles in the fact that it is in these paretic aural affections that galvanism will be found of essential service. That is to say, it is in the treatment of deafness in adult life, when this acknowledges a more deeply scated constitutional lesion of the nervous system, that the true province of electro-therapeutics, as it relates to otology, will be found to lie. Speaking strictly within the limits of my own experience, I have to regret that, even in this large class of cases, the use of electricity is essentially a limited one. The reason of this resides in the fact that we seldom meet with uncomplicated cases; for it is inherent in the nature of any affection which interferes with the function of the Eustaclian tube-as paralysis of its muscles does-to induce a greater or less degree of passive congestion of the middle ear. Now it has been cunclusively shown by the experiments of Morant and Destre, a detailed account of which will be found in the Comptes Rindas, $18 \% 8$, that, after galvanising motor nerves, there follows considerable hypermmin in the tissues to which the branches are distributel, which condition 
will endure for some days. This effect is due to the circumstance that searly ali such nerves contain vaso-motor fibrille, and is owing to the dilating influence of the galvanism on the vessels to which these vasomotor tilaments are ultimately distributed. Therefore, while the application of the electric current will tend to restore the motor power of the muscles to which it is applied, it will at the same time perpetuate, if not increase, the already existing congestion of the middle ear.

This, I take it, is the reason why deaf patients subjected to the trcatment in question fail to afford results proportionate to the restoration which we know has accrued in the muscle-power of the tube in consequence of their successful electrisation.

Obviously, therefore, the question of the use of electricity in a given case of ear-disease is one not to be decided without due deliberation and the exercise of considerable discrimination. These are points on which, doubtless, much information will be elicited from the discussion of the subject. Other points, respecting which the experience of cach yractitioner will give valuable hints, are, the kind of current suitable to the case to be treated, whether the continuous, the interrupted, or the induced current; also the strength of the current and the frequency of application. Speaking on these points from my own experience, I may say that I have nearly abandoned the constant battery for therapeutic purposes, and use most frequently a very weak induced current, and never repeat the application oftener than once a week.

Respecting the instruments necessary for the purpose, the simpler they are the better, providing efficiency be not sacrificed to obtain this end.

Cralvanisation of the tensor tympani muscle with certainty can be best accomplished by means of Weber-Liel's intratubal electrode. It consists of a delicate gum-elastic catheter, which is introduced into the Eustachian tube to the desired length through an ordinary catheter. Through it runs a platinum wire, which is exposed for a short space near its distal end by means of an opening at the side of the elastic tubs. The near end of this wire is attached to a ring, to which the cord communicating with the battery is hooked. The other pole can be applier by means of a sponge-holder either to the mastoid process or an indifferent part.

It has been objected by Burnett, that the benefit resulting from the above procedure arises from the mechanical effect of the intratubal electroile acting as a bougie, rather than to the electricity applied through it means. Practically, I have found some difficulty in introducing Weber-Liel's instrument, from its exceeding pliability. Others, however, may have been more successful.

For galvanising the extratubal muscles I have long used a very simple electrode, consisting of an ordinary vulcanite catheter tipped with a metal rim, to which, inside and isolated by the vulcanite tube, is at ached a wire, which at the opposite end joins a ring for connection with the battery. With this the remote portions of the tensor polati can be readily reached. The circuit is completed by the spongeholder applied to the mastoid process. I have occasionally got very crood results from this arrangement as regards increase of hearing power; and, as there can be no question of this instrument acting as a bougie, for it certainly does not get beyond the mouth of the tube, such improvement may fairly be attributed to the influence of the electric current. So far as this fact goes, it would seem to weaken Burnett's objection to Weber-Liel's instrument.

Looking to the important part which the tensor palati muscle plays in securing the functions of the Eustachian tube, it being the principal rilator of this canal; and seeing that the section of this muscle after leaving the hamular process is readily accessible on the anterior surface of the soft palate; and seeing that it is chiefly to paresis of this muscle that those objective signs of paresis of the palate are due, to which reference has been made,-it is my frequent practice to galvanise this inuscle through the mouth-a proceeding which is very easy of performance. For this purpose, I use an ordinary laryngeal electrode, but somewhat larger than this instrument is now made; applying it over the soft palate in the course taken by the muscle on each side of the uvula, the circuit being completed by placing the sponge-holder attached to the other pole over the mastoid process of that side corresponding to the side of the palate to which the laryngeal electrode is being used.

Ar: the last sitting of the select council on the Artisans Dwellings' Act, the medical officer of the parish of St. George's-in-the-East, stated that in a district between the London Docks and the Thames there were houses, occupied by the lowest form of labourers, with only two rooms, in which as many as twelve persons were living. In others there were six in two rooms of about 750 cubic feet each. The death-rate in the wirst part of this district was thirty-one per 1,000 , and the rate all over the district was 25 per I,000.

\section{TWO CASES OF STRANGULATED HERNIA: WITH SOME NOTES ON THE OPERATION.*}

By PAUL W. SWAIN, F.R.C.S.,

Surgeon to the South Devon and East Cornwall Hospital, Plymouth.

I HAve selected strangulated hernia as the subject for a paper, partly because, within the last fortnight, two cases presenting some peculiarities have occurred in my practice, and partly because it is a subject of such universal interest, that few members of our Branch can fail to contribute material information during the discussion which, I trust, will follow the reading of this paper.

On December 22nd, I was asked by Messrs. Miles and Stamp of Plympton to see a lady, aged 55 , with symptoms of strangulated femoral hernia. On the previous day, she had been quite well up to the time of her late dinner, when, after a fit of coughing, she was seized with sudden pain in the abdomen, and vomiting. She also had an idea that, shortly before, she had strained herself by lifting a heavy musicbook. She was seen at once by Mr. Stamp, who found that she had a tense and painful swelling in her left groin, which he naturally conclucled to be a hernial protrusion. $U_{p}$ to this point, the case seemed clear; but here came in a bit of previous history, which at once introduced an element of grave doubt. Two years ago, this lady had been engaged in selling at a bazaar, and had been standing about more or less all day. The next day, she complained of pain and swelling in her left groin, accompanied with sickness. The medical man, under whose care she then was, considered that the swelling was due to an enlarged gland; and she was treated for some weeks by rest and iodine. As to whether the swelling had ever entirely subsided from that time to the present, there was no clear history. I arrived on December 22nd, at 5.30 1'M., just twenty-four hours after the primary attack of pain and vomiting. Her pulse and temperature were both normal, her countenance tranquil, and her tongue moist but furred. She had no abdominal tension or tenderness. There had been an evacuation from the bowels that morning after an enema. Twice or thrice during the day she had vomited green bilious matter, with no suspicion of stercoraceous vomit. The swelling in her left groin was about the size of a small hen's-egg, very tense, somewhat painful on pressure, and decidedly resonant on percus. sion. I had not the slightest doubt in my own mind that it was a hernial protrusion. Looking, however, to her previous history, and at the same time taking into consideration the absence of urgent symptoms, we determined to violate one of the golden rules of surgery, and to wait. We, therefore, decided on giving a quarter of a grain of extract of belladonna, every four hours; to apply ice to the swelling; and to feed her on small quantities of lime-water and milk, and Brand's essence of beef. She was also to have another simple enema. I heard no more of the case for the next forty-eight hours, when I was again requested to see her. The change in her condition, although not great, was very decidedly for the worse. She had had no motion from the bowels; the sickness of the same character was still present. Her countenance was somewhat more anxious, and the abdomen rather tense. No further delay was admissible; and, the patient consenting, the operation was performed under the influence of methylene. You will doubtless agree with me that no two operations for the relief of strangulated hernia are ever alike, and this one did not fail to have its peculiarity. We are taught in the books that, when fluid escapes upon puncturing one of the layers of tissue, you may feel confident that you have entered the sac of the hernia; and, as a rule, doubtless this is the case. But, on the present occasion, I divided three successive layers of tissue, from under each of which a large quantity of fluid escaped. The last one was the sac itself, and within it there was no difficulty in recognising the knuckle of intestine. The difference in colour is so striking that a mistake can hardly be made. The sac is of a pearly white colour, whereas the intestine varies from bright rose to coal-black, according as it has been strangulated a shorter or a longer time. For the rest, I may say that the patient made a perfect recovery, without a bad sym. ptom - the wound healing by the first intention, and that without the intervention of Listerism.

You will naturally ask: Why, if you were convinced you had to deal with a constricted knuckle of intestine, did you wait? My answer is, that $I$ had in my mind an acute recollection of a very instructive case, which happened to a colleague of mine, in the practice of the Royal Albert Hospital. A woman whom I myself sent into hospital, with what I thought to be a strangulated femoral hernia, fell to the lot of my colleague (Mr. Bulteel), who, agreeing with me, operated; and, having removed an enlarged gland, could find no sign of intestine. Upon 